\title{
Nephropathy due to Aminoglycosides
}

National Cancer Institute

\section{Source}

National Cancer Institute. Nephropathy due to Aminoglycosides. NCI Thesaurus. Code C123132.

Kidney damage resulting from aminoglycosides. 